form expressions for the polynomials for the triangle, for example, are believed not to have been known previously.

Certain properties of the polynomials of an orthogonal system for which expressions in closed form exist can be derived easily. This, in fact, motivates the search for them. For instance, for all the systems obtained above the generating function can be obtained by means of Lagrange's formula. For the unit circle the work is outlined in [2].

\title{
REFERENCES
}

1. W. Groebner, Ueber die Konstruktion von Systemen orthogonaler Polynome in ein- und zwei-dimensionalen Bereichen, Monatsh. Math. vol. 52 (1948) pp. 38-54.

2. F. Didon, Développements sur certaines séries de polynomes a un nombre quelconque de variables, Ann. Sci. Ecole Norm. Sup. vol. 7 (1870) pp. 247-268.

Michigan State University

\section{A COUNTER-EXAMPLE TO A STATEMENT ON CON- FORMAL MAPPING OF RIEMANN SURFACES}

\author{
JAMES A. JENKINS ${ }^{1}$
}

A few years ago H. L. Royden [3] asserted a result which in the nomenclature of the author [2] is stated as follows.

Let $\Re$ be a finite oriented Riemann surface, $Q(z) d z^{2}$ a positive quadratic differential on $\Re, \Delta$ an admissible domain with respect to $Q(z) d z^{2}$. Then the identity is the only conformal mapping $f$ of $\Delta$ into $\Re$ which has all the following properties:

(i) there is an interior point of $\Delta$ which is a fixed point of $f$;

(ii) the poles of $Q(z) d z^{2}$ which lie in $\Delta$ are fixed points of $f$ and near a pole of order $k \geqq 2$ the mapping has the form

$$
f(z)=z+a z^{k}+\cdots
$$

in terms of a local uniformizing parameter $z$ at the pole;

(iii) $f$ admits an admissible homotopy into the identity.

We will give now a simple example to show this assertion incorrect.

Let us denote by $\alpha$ the quantity $\left(e^{2 \pi} /\left(e^{2 \pi}-1\right)\right)^{1 / 2}$ and by $D$ the domain on the $z$-sphere exterior to the circle $|z-\alpha|=1$ (i.e., containing the point at infinity). Let $f_{\theta}(z)$ be the function defined in $[2$,

Received by the editors October 17, 1958.

${ }^{1}$ Research supported in part by the National Science Foundation, Mathematics Section, through the University of Notre Dame and by the Office of Ordnance Research under contract No. DA-36-034-ORD-2453 through the Institute for Advanced Study. 
Theorem 5.3] mapping $D$ onto a domain admissible with respect to the quadratic differential $e^{-2 i \theta} d w^{2} / w^{2}$. Then from [2, Corollary 6.12] we see that

$$
f_{\pi / 4}^{\prime}(0)=1 \text {. }
$$

If then $\Delta=f_{\pi / 4}(D)$ and $\phi(w)$ denotes the function inverse to $f_{\pi / 4}(z)$ defined in $\Delta$ then $\phi$ possesses all the properties assumed by Royden yet is not the identity. It is evident that many other similar examples may be given.

Royden's result fails where the General Coefficient Theorem succeeds primarily because he failed to recognize the essential role played by the concept of deformation degree. One error in his purported proof certainly lies in the incorrectness of his Lemma 1 as has been pointed out elsewhere $[2$, p. 71$]$.

In the case where no poles of order at least two are present the above result was essentially proved by Teichmüller [4].

\section{BIBLIOGRAPHY}

1. James A. Jenkins, A general coefficient theorem, Trans. Amer. Math. Soc. vol. 77 (1954) pp. 262-280.

2. - Univalent functions and conformal mapping, Springer-Verlag, BerlinGöttingen-Heidelberg, 1958.

3. H. L. Royden, The conformal rigidity of certain subdomains on a Riemann surface, Trans. Amer. Math. Soc. vol. 76 (1954) pp. 14-25.

4. O. Teichmüller, Extremale quasikonforme Abbildungen und quadratische Differentiale, Abhandlungen der Preussischen Akademie der Wissenschaften, Mathematisch-naturwissenschaftliche Klasse, 1939, No. 22.

Institute For Advanced Study AND

UNIVERSITY OF Notre DAME 